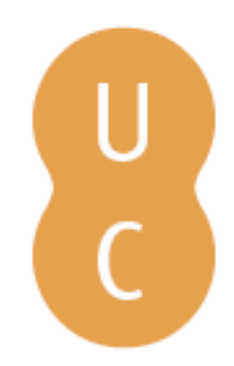

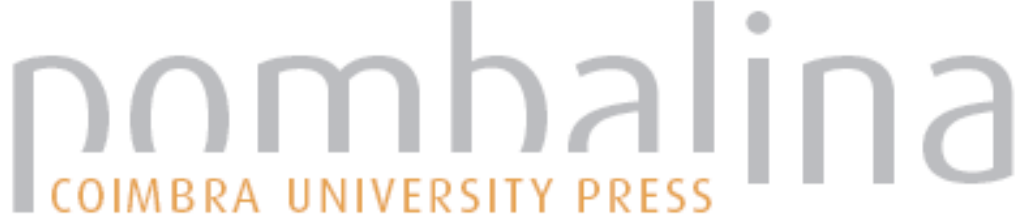

\section{O vinho no bem-estar da mesa e do corpo}
Autor(es): Seiça, Raquel
Publicado por: Imprensa da Universidade de Coimbra
URL
persistente: URI:http://hdl.handle.net/10316.2/45235
DOI: $\quad$ DOl:https://doi.org/10.14195/978-989-26-1721-3_8

Accessed : $\quad$ 26-Apr-2023 12:46:11

A navegação consulta e descarregamento dos títulos inseridos nas Bibliotecas Digitais UC Digitalis, UC Pombalina e UC Impactum, pressupõem a aceitação plena e sem reservas dos Termos e Condições de Uso destas Bibliotecas Digitais, disponíveis em https://digitalis.uc.pt/pt-pt/termos.

Conforme exposto nos referidos Termos e Condições de Uso, o descarregamento de títulos de acesso restrito requer uma licença válida de autorização devendo o utilizador aceder ao(s) documento(s) a partir de um endereço de IP da instituição detentora da supramencionada licença.

Ao utilizador é apenas permitido o descarregamento para uso pessoal, pelo que o emprego do(s) título(s) descarregado(s) para outro fim, designadamente comercial, carece de autorização do respetivo autor ou editor da obra.

Na medida em que todas as obras da UC Digitalis se encontram protegidas pelo Código do Direito de Autor e Direitos Conexos e demais legislação aplicável, toda a cópia, parcial ou total, deste documento, nos casos em que é legalmente admitida, deverá conter ou fazer-se acompanhar por este aviso.

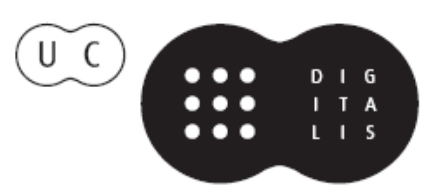


Carmen Soares

Gilene da Silva Gomes Ribeiro

(coords.)

\section{Mesas \\ LUSO-BRASILEIRAS}

\section{ALIMENTAÇÃO, SAÚdE \& GULTURA}

\section{VOLUME I}

IMPRENSA DA UNIVERSIDADE DE COIMBRA COIMBRA UNIVERSITY PRESS

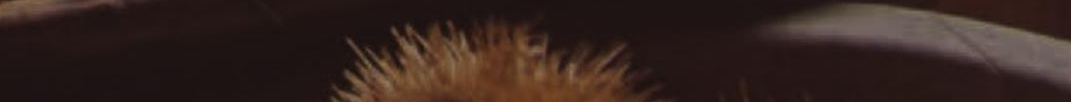




\title{
O VINHO NO BEM-ESTAR DA MESA E DO CORPO
}

\section{(The role of wine in the meal and body welfare)}

\author{
Raquel Seiça \\ Universidade de Coimbra, Faculdade de Medicina, Instituto de \\ Fisiologia e Instituto de Imagem Biomédica e Ciências da Vida (IBILI) \\ Centro de Estudos Clássicos e Humanísticos da Universidade de Coimbra \\ Projeto DIAITA: Património Alimentar da Lusofonia \\ (rmfseica@gmail.com)
}

Resumo: O consumo do vinho faz parte da cultura de muitos povos desde a Préhistória. O vinho foi tóxico, mas igualmente prazer e relaxante, com influências no humor e na sociabilidade. Desde a Antiguidade, o vinho apresenta-se, também, intimamente ligado à medicina sendo uma das mais antigas prescrições médicas, um remédio para o corpo e para a alma. No século passado, foram reconhecidos os malefícios do consumo abusivo de vinho, risco acrescido para o desenvolvimento de várias doenças, para o aumento da mortalidade por acidentes de viação e de trabalho e para diversos problemas sociais. Nas duas últimas décadas, a investigação clínica e epidemiológica restabelece o seu poder medicinal, particularmente do vinho tinto a quem são atribuídos efeitos anti-oxidantes, anti-inflamatórios e anti-aterogénicos. O vinho, particularmente o vinho tinto, consumido de forma moderada, regular e à refeição, é parte integrante da dieta mediterrânica que se associa a benefícios vários nomeadamente nas doenças cardiovasculares e na diabetes. Consumir adequadamente vinho, particularmente o tinto, alimenta o prazer e pode cuidar do corpo e aumentar a longevidade. Tal como outrora, consumir vinho moderadamente ou de forma excessiva pode significar a diferença entre remédio e veneno, entre prevenir e aumentar a mortalidade.

Palavras-chave: vinho, polifenóis, dieta mediterrânica, saúde, doenças cardiovasculares.

Aвstract: The consumption of wine is part of several people cultures since Prehistory. The wine was considered to be toxic but also an enjoyment and a relaxant, acting on the humour and the conviviality. Since the Ancient times the wine is closely linked to medicine being one of the oldest medicine prescription, a drug for body and soul. The adverse effects of wine abuse were recognized in last century, a risk for several disease development and increased mortality by traffic and professional accidents and social problems. In the last twenty years, clinical and epidemiological studies restored its medicinal properties, namely of red wine 
in relation to its antioxidant, anti-inflammatory and anti-atherogenic effects. The moderate consumption of wine, particularly red one, drinked regularly in the meal, is a component of mediterranean diet which has health benefits namely in cardiovascular diseases and diabetes. An appropriate wine consumption, specifically the red wine, feed the pleasure and may care the body and increase longevity. As in Ancient times, moderate or excessive wine consumption can mean the difference between drug and poison, between prevent and increase mortality.

KEYWORDs: wine, polyphenols, mediterranean diet, health, cardiovascular diseases.

O consumo do vinho, que vem da Pré-história, foi usado por diferentes razões mas não se conhecem os detalhes de então sobre o cultivo das videiras e a fermentação do vinho.

Muito da cultura actual do vinho provém, sim, das práticas da Grécia Antiga e do Império Romano, com um enorme impacto no desenvolvimento da viticultura e da enologia.

O vinho, a bebida mais prestigiada dos povos da Grécia e da Roma Antigas, foi utilizado isoladamente, considerado dessa forma um redutor do espírito e um causador de intoxicações ou loucura, e diluído em água ou com aditivos vários, como ervas aromáticas e mel. Assumiu-se, dessa forma, como elo de relacionamento interpessoal, uma arte de bem-receber, uma presença nos jantares/banquetes dos mais ricos e nos encontros de discussão e troca de saberes ${ }^{1}$. O vinho foi prazer, foi relaxante, com influências no humor e na sociabilidade. $\mathrm{O}$ seu valor hedónico é desde sempre reconhecido; são as sensações agradáveis que desperta quando se olha, se cheira, se prova. Arraigado na cultura greco-latina, o vinho assume-se, ainda hoje, como um dos componentes principais da dieta mediterrânica ${ }^{2}$.

$\mathrm{Na}$ cozinha medieval, o vinho manteve a sua presença, frequentemente diluído em água, sendo a bebida comum de todas as classes sociais no sul da Europa. A falta de segurança no consumo da água terá sido uma das razões para a popularidade do vinho nesta época.

$\mathrm{O}$ vinho teve igualmente um significado religioso, nomeadamente na liturgia cristã, servindo aqui um papel essencial na celebração da missa. A Igreja Católica teve ainda um papel crucial na preservação da viticultura e da enologia, sendo de realçar o papel dos monges Franciscanos, Beneditinos e Cistercienses ${ }^{3}$.

Desde a Antiguidade, o vinho apresenta-se, também, intimamente ligado à medicina sendo uma das mais antigas prescrições médicas, um remédio

\footnotetext{
${ }^{1}$ Soares 2014: 17-50; Padrões Culturais Editora 2005: 9-26; Dalby 2003: 350-352.

2 A este propósito leia-se Soares 2014: 17-50.

3 Padrões Culturais Editora 2005: 9-26.
} 
para o corpo e para a alma. Durante séculos, beber vinho foi tido como um benefício para a saúde, com efeitos no bem-estar físico e emocional, pelo que são inúmeros os relatos históricos recomendando esse hábito.

Os gregos consumiam o vinho integrado na sua dieta e como remédio ou meio de toma de medicamentos. Era desinfectante de feridas e meio de imersão de diversas ervas. $\mathrm{O}$ vinho ocupou, de facto, um papel central na medicina da época. Hipócrates (460-370 a.C.), o grande médico grego considerado o pai da medicina, considerava o vinho como parte integrante de uma alimentação saudável. Incorporou o vinho no tratamento de doenças agudas e crónicas, recomendando o seu uso como suplemento dietético, pelo seu poder digestivo, antisséptico e diurético e como meio de mistura de drogas para administração aos doentes. A sua escola médica foi a primeira a usar o vinho como medicamento ${ }^{4}$.

Também Galeno (129-216 d.C.), um dos maiores médicos depois de Hipócrates, deu o seu particular contributo à utilização do vinho como veículo na preparação de remédios vegetais (formulações galénicas). Deixou-nos um tratado "Dos antídotos" sobre a utilização de preparações de vinho e ervas como antídotos de venenos, e com descrições perfeitas sobre os vinhos bebidos na época em Roma, como eles deveriam ser analisados, guardados e envelhecidos.

No século XIV surge o primeiro livro impresso e talvez o mais importante depois dos escritos de Galeno, "Liber de Vinis", da autoria do médico e professor da Universidade de Montpellier, Arnaldo de Villanova. Nele são citadas as propriedades curativas de vinhos aromatizados com ervas e a sua aplicação numa enorme diversidade de doenças.

Destacam-se, mais tarde, a Farmacopeia de Londres (1618) e a Francesa (1840) com numerosas misturas contendo vinho. Do século XVII ao século XIX o vinho foi prescrito como estimulador do apetite, diurético, sedativo e, acima de tudo, um agente promotor de bem-estar físico e emocional.

Relevante ainda, no século XIX, as descobertas de Louis de Pasteur sobre os microorganismos e a fermentação, publicadas no seu livro Études sur le Vin-ses maladies, causes qui les provoquent, procédés nouveaux pour le conserver et pour le vieillir, que constituem uma referência no campo da enologia moderna ${ }^{5}$.

\footnotetext{
4 Padrões Culturais Editora 2005: 9-26.

5 Padrões Culturais Editora 2005: 9-26.
} 


\section{Vinho e Saúde}

O vinho, como já referido, é um elemento da cultura de muitos povos, desde o início da história da humanidade, e também da história da medicina sendo-lhe atribuídos efeitos opostos no organismo, tóxico ou benéfico, na dependência de como é utilizado.

No século passado houve drásticas mudanças na sociedade e na medicina face ao consumo de álcool e, como tal, do vinho. Foram reconhecidos os malefícios da ingestão abusiva de bebidas alcoólicas (alcoolismo), risco acrescido para o desenvolvimento de doenças hepáticas, nomeadamente a cirrose hepática alcoólica, mas também hipertensão arterial, cardiomiopatia alcoólica, arritmia auricular e doença aterotrombótica, especialmente a doença coronária, alterações nervosas como deficiência cognitiva e demência, infertilidade e cancro, nomeadamente do cólon e do recto, da mama, da laringe e do fígado. Mais, o consumo de bebidas alcoólicas, exagerado e fora das refeições, assumiu um papel de relevo no aumento da mortalidade por acidentes de viação e de trabalho e ainda em diversos problemas sociais, profissionais e familiares com redução das capacidades de trabalho e incumprimento da actividade profissional e comportamentos familiares e sociais desequilibrados ${ }^{6}$.

Mas nas duas últimas décadas, a investigação clínica e epidemiológica restabelece o seu poder medicinal. Atribuía-se uma variedade de benefícios ao consumo de bebidas fermentadas como o vinho e a cerveja, embora sem qualquer base científica. Aqueles estudos vieram demonstrar que o consumo de vinho, diário e à refeição (e não esporádico e excessivo) e em dose baixa-moderada (1 copo por dia na mulher e 1 a 2 copos por dia no homem), tem benefícios vários, com redução da morbilidade e da mortalidade maioritariamente associadas a doenças cardiovasculares. De facto, esta forma de beber vinho parece reduzir a incidência de doenças cérebro e cardiovasculares e doenças neurodegenerativas (o consumo excessivo causa lesões cerebrais mas o consumo moderado do vinho tinto parece retardar o declínio das funções cognitivas) bem como a incidência de hipertensão arterial, diabetes mellitus (redução do risco de desenvolvimento de diabetes tipo 2 e relação inversa com as complicações microvasculares da diabetes tipo 1 , retinopatia, neuropatia e nefropatia) e alguns tipos de cancro (cólon, ovário, próstata), estando ainda implicado no aumento da longevidade ${ }^{7}$.

O efeito benéfico do consumo baixo-moderado de vinho no cancro é, contudo, controverso embora estudos in vitro apontem para o efeito anticancerígeno dos polifenóis (componentes do vinho, assunto desenvolvido mais adiante), que contrariam os malefícios do etanol, considerado um agente

\footnotetext{
${ }^{6}$ Arranz 2012: 759-781; Klatsky 2015: 238-250; O'Keefe 2014: 382-393.

Arranz 2012: 759-781; Artero 2015: 3-13; O’Keefe 2014: 382-393; Cueva 2017: 99.
} 
carcinogénico. $\mathrm{O}$ impacto do vinho na longevidade tem igualmente suscitado controvérsia; alguns estudos apontam para o efeito do vinho na longevidade, não observado com a cerveja e os vinhos espirituosos, sendo ainda conflituoso o efeito preferencial do vinho tinto em relação ao vinho branco ${ }^{8}$.

Apesar das dúvidas, dos resultados conflituosos, as evidências suportam a hipótese de que o consumo baixo-moderado de vinho, atendendo às suas propriedades preventivas e terapêuticas em diversas doenças, pode prolongar a vida saudável, sobretudo o vinho tinto dadas as propriedades anti-inflamatórias ${ }^{9}$, anti-oxidantes ${ }^{10}$ e anti-aterogénicas ${ }^{11}$ dos seus componentes.

O consumo moderado de cerveja tem também sido associado a estes efeitos mas em menor grau provavelmente devido ao menor conteúdo em polifenóis. Mantém-se a controvérsia sobre os efeitos das diferentes bebidas na saúde e na longevidade, não só do vinho e da cerveja mas também das bebidas espirituosas, nomeadamente no sistema cardiovascular e no cancro, e também se esses efeitos devem ser atribuídos ao etanol ou aos restantes componentes das bebidas, nomeadamente os polifenóis.

Cabe-nos fazer, aqui, uma referência à composição do vinho. Esta é afectada por vários factores incluindo a variedade das uvas, a tecnologia de fabrico do vinho e as suas condições de armazenamento e envelhecimento. Mas, mesmo considerando estes factores, o vinho tem na sua constituição água, etanol, glicerol, polissacarídeos, diferentes tipos de ácidos e compostos fenólicos ou polifenóis sendo estes flavonóides e não-flavonóides.

O vinho tinto resulta da fermentação do sumo da uva na presença da casca e das sementes, ambas contendo a maior parte dos polifenóis, enquanto o vinho branco é um produto do sumo, pelo que o vinho tinto pode conter dez vezes mais compostos fenólicos que o vinho branco. Os flavonóides, que correspondem a cerca de $85 \%$ dos polifenóis do vinho tinto, incluem os

8 Arranz 2012: 759-781; Artero 2015: 3-13.

${ }^{9}$ A inflamação faz parte da resposta protectora do organismo face a agentes agressores. Mas a inflamação prolongada, crónica conduz a alterações celulares progressivas estando pois implicada no desenvolvimento de várias doenças crónicas como a aterosclerose, o cancro, a diabetes e a obesidade. Combater este processo através de substâncias anti-inflamatórias pode reduzir a progressão destas doenças.

${ }_{10}$ As reacções de oxidação que ocorrem no organismo, podem produzir radicais livres que, por sua vez, podem dar início a reacções em cadeia danificando células ou causando a sua morte. Os anti-oxidantes interrompem estas reacções eliminando os radicais livres e inibindo outras reacções de oxidação. O organismo tem defesas anti-oxidantes enzimáticas e não enzimáticas que controlam a agressão oxidativa. O desequilíbrio entre a produção e a eliminação de radicais livres, resultante da redução destas defesas ou do aumento da produção de radicais livres, causam stress oxidativo, um processo subjacente ao desenvolvimento de várias doenças nomeadamente a diabetes e doenças cardiovasculares e neurodegenerativas.

11 A aterogénese diz respeito à formação de placas de ateroma na parede arterial, condição essencial na patogenia da aterosclerose. As substâncias anti-aterogénicas podem prevenir ou inibir este processo. 
flavonóis, de que é exemplo a quercetina, as flavonas e as antocianinas. Os não-flavonóides incluem ácidos e estilbenos, e o seu derivado resveratrol, e as taninas ${ }^{12}$.

Os polifenóis são fitonutrientes, compostos naturais aos quais se tem atribuído propriedades biológicas com acções preventivas e terapêuticas numa variedade de doenças. A maior fonte desses compostos encontra-se na fruta e em bebidas como a cerveja, o chá verde e particularmente o vinho. O teor de polifenóis pode ser afectado por factores ambientais (humidade, exposição solar, características do solo) e agrários como o tipo de cultura, bem como pelo armazenamento e processamento dos alimentos e bebidas. Mais ainda, os polifenóis mais comuns da dieta humana podem não ser necessariamente os mais activos no organismo podendo ter antes uma reduzida actividade intrínseca, baixa absorção intestinal e metabolismo e eliminação mais rápidos ${ }^{13}$. De facto, estima-se que somente $5-10 \%$ dos polifenóis da dieta sejam absorvidos no intestino delgado e $90-95 \%$ atinjam o cólon onde são convertidos, pela microbiota intestina $1^{14}$, em metabolitos que poderão ser biologicamente mais activos que os seus precursores actuando a nível local e sistémico ${ }^{15}$.

O principal constituinte activo de qualquer bebida alcoólica é o etanol que se assume como um tóxico e como um benefício para a saúde. Embora não esteja completamente esclarecido se uma bebida induz maior proteç̧ão cardiovascular que outra, a maior parte dos estudos sugere que os componentes não-etanol, sobretudo os polifenóis presentes nas bebidas fermentadas, vinho e cerveja, contribuem largamente para os benefícios cardiovasculares tendo sido sugerido que são sobretudo os constituintes não-etanol, e não o etanol, que previnem ou contrariam a disfunção do endotélio vascular ${ }^{16}$ induzida pela dieta ocidenta ${ }^{17}$.

O vinho, particularmente o tinto, dada a sua riqueza em compostos não-etanol, tem sido associado a maiores benefícios na saúde. Estudos in vitro e em modelos animais apontam para o papel benéfico dos componentes não-etanol do vinho, particularmente os compostos fenólicos do vinho tinto, consequência das propriedades anti-inflamatórias, anti-oxidantes, e

12 Artero 2015: 3-13.

13 Arranz 2012: 759-781; Artero 2015: 3-13.

${ }^{14}$ A microbiota intestinal diz respeito à complexa população de microorganismos residente no intestino humano.

15 Cueva 2017: 99.

16 As células do endotélio vascular têm importantes funções na homeostasia dos vasos sanguíneos. A disfunção destas células ou seja, a perda das suas funções próprias, associa-se a uma série de condições fisiopatológicas como a aterosclerose, a hipertensão arterial e a diabetes.

17 O’Keefe 2014: 382-393; Vilahur 2014: 1177-1188. 
anti-aterogénicas anteriormente mencionadas. Mas os efeitos no Homem não estão ainda devidamente estabelecidos.

Os mecanismos responsáveis por estes efeitos envolvem diferentes vias sendo ainda controversa, como já dito, a qual dos componentes, etanol e compostos fenólicos, se devem atribuir. Os mecanismos de acção na aterosclerose envolvem a regulação lipídica e os efeitos anti-inflamatório e anti-oxidante. $\mathrm{O}$ etanol aumenta o colesterol-HDL, inibe a agregação plaquetar e reduz a inflamação sistémica, podendo assim contribuir para a redução da aterogénese. Contudo, o vinho tinto providencia benefícios adicionais relativamente às outras bebidas alcoólicas, provavelmente devido ao conteúdo fenólico, como redução da pressão arterial, inibição da oxidação das lipoproteínas LDL, melhoria da função endotelial e redução da inflamação e da adesão celular, contributos na prevenção e tratamento de várias doenças como as doenças cardiovasculares ${ }^{18}$.

Em relação aos compostos fenólicos, tem sido dada uma atenção particular ao resveratrol, um polifenol não flavonóide. O resveratrol é produzido naturalmente na casca da uva e nas sementes, durante a fermentação. No processo de produção do vinho branco, há um menor contacto com a casca da uva e as sementes e, como tal, menor conteúdo em resveratrol. Ao resveratrol são atribuídas propriedades anti-oxidantes, anti-inflamatórias e anticancerígenas e efeitos protectores cardiovasculares e neurológicos e no aumento da longevidade. De facto, foi demonstrado o efeito do resveratrol na prevenção de doenças crónicas como doenças cardiovasculares e diabetes mellitus, doença de Alzheimer e alguns tipos de cancro ${ }^{19}$.

O resveratrol tem sido, pois, considerado o constituinte funcional major do vinho tinto, responsável por prevenir ou retardar uma variedade de doenças e aumentar a longevidade. Contudo, comparado com os restantes compostos fenólicos, a concentração de resveratrol no vinho tinto é baixa pelo que se questiona hoje o seu papel central nos efeitos benéficos do vinho tinto, particularmente a actividade anti-oxidante que parece dever-se ao conjunto dos polifenóis e não exclusivamente ao resveratrol ${ }^{20}$. A esse propósito, entre os flavonóides, a quercetina tem sido um alvo particular e sobretudo pelas suas actividades anti-oxidantes e anti-inflamatórias ${ }^{21}$.

Os efeitos benéficos do vinho devem ser antes atribuídos ao conjunto de todos os seus constituintes e não a um componente específico.

O binómio vinho e saúde é, pois, algo ainda controverso e alvo de investigação. Compreender a resposta biológica ao vinho é limitada, por

\footnotetext{
18 Arranz 2012: 759-781.

19 Artero 2015: 3-13.

${ }^{20}$ Xiang 2014: 258-263.

21 Artero 2015: 3-13.
} 
um lado, pela variedade dos seus constituintes bioactivos e, por outro, pela multiplicidade de mecanismos de acção de cada componente. Também a proporção dos seus constituintes depende da variedade das uvas, da região do cultivo, do método de produção e do processo de envelhecimento do vinho.

Investiga-se a diferença entre as diversas bebidas, vinho, cerveja e vinhos espirituosos (estes têm essencialmente etanol) e o papel dos seus diferentes constituintes, sobressaindo, nos estudos efectuados, a importância dos compostos não etanol, especificamente os polifenóis, como já referido.

São necessários estudos adicionais no Homem de forma a reforçar a evidência experimental respeitante aos efeitos benéficos da ingestão de vinho. Mas, apesar das controvérsias, sugere-se que o vinho, especialmente o tinto, tem efeitos positivos no organismo, particularmente nas doenças cardiovasculares. Mesmo considerando todas as limitações, podemos afirmar que o consumo baixo-moderado de vinho, particularmente o tinto, pode ter efeitos benéficos na saúde.

\section{O Vinho na Alimentação - o vinho na dieta mediterrânica}

No campo da medicina, a alimentação tornou-se um dos mais importantes aspectos da saúde e do bem-estar do indivíduo, tendo a dieta um papel fulcral no desenvolvimento de várias doenças crónicas. Os hábitos alimentares e de vida são factores particularmente dependentes do comportamento das pessoas e têm repercussões múltiplas na saúde. A alimentação tem pois um enorme impacto; de certa forma, nós somos o que comemos e a nossa saúde está profundamente ligada ao que comemos diariamente.

A alimentação é um meio de sobrevivência tendo o organismo mecanismos fisiológicos que regulam o comportamento alimentar e a homeostasia energética. Nos dias de hoje, com a disponibilidade de alimentos saborosos, ricos em gorduras e açúcar, o seu consumo passou a ser um prazer e não somente uma necessidade calórica, energética, de forma a manter a homeostasia. É a fome hedónica contrariando a fome homeostática. Esta tem também um componente hedónico, mas o corpo serve-se de mecanismos de retrocontrolo negativo. $\mathrm{Na}$ fome hedónica não se come em resposta a um estado de deplecção energética; come-se unicamente pelo valor gustativo e pelas propriedades de recompensa, de forma independente do conteúdo calórico. $\mathrm{Na}$ alimentação homeostática a deficiência energética leva à activação de mediadores hipotalâmicos que promovem a fome e a ingestão de alimentos a que se segue a libertação de mediadores da saciedade conducente à paragem do consumo de mais alimentos. Já na alimentação hedónica, os alimentos saborosos actuam em circuitos cerebrais de recompensa que libertam mediadores, como a dopamina, os endocanabinóides e os opiáceos, responsáveis pela estimulação persistente dos centros hipotalâmicos da fome e pela inibição dos mediadores 
da saciedade. Assim, o impulso de comer mantém-se e os alimentos são consumidos não pelas necessidades energéticas, mas em consequência das suas propriedades de recompensa e de prazer.

Em relação ao consumo de alimentos menos saudáveis, surge-nos, em oposição, a dieta mediterrânica, uma forma saudável de estar na vida, um modelo dietético e cultural, um conjunto de tradições, conhecimentos e competências que passam de geração em geração ${ }^{22}$. O seu sucesso relaciona-se com o efeito dos seus constituintes e com o ambiente tradicional e social que a envolve. De facto, viver de forma mediterrânica significa não somente alimentação saudável, mas também uma forma específica de vida, feita de convivialidade e tradições. A dieta mediterrânica, reconhecida como Património Cultural Imaterial da Humanidade, não é somente um conjunto de alimentos específicos, diversos e que variam consoante a época do ano. Envolve a convivialidade à mesa, um momento familiar, social e de comunicação; valoriza a hospitalidade, reúne pessoas de todas as idades e classes sociais, assumindo um papel fulcral em eventos culturais e festivos e celebrações várias.

A tradição mediterrânica oferece uma cozinha rica em cores, aromas e memórias que suportam o gosto dos povos mediterrânicos. A dieta mediterrânica tem a sua origem na bacia mediterrânica, para muitos o berço da sociedade, sendo os gregos os criadores e difusores de um padrão alimentar característico que tem como tríade o azeite, o pão e o vinho, igualmente elementos centrais da liturgia cristã. É um modelo nutricional universalmente apreciado, uma pertença social, histórica, territorial e ambiental, intimamente ligada ao estilo de vida dos povos mediterrânicos ${ }^{23}$.

A dieta mediterrânica é hoje um modelo de "comer saudável" baseado na sua relação com a preservação do bom estado de saúde e da qualidade de vida tendo como características major o elevado consumo de frutos, verduras e legumes, nozes, cereais e peixe, o consumo de azeite como gordura principal, o consumo moderado de lacticínios, mais sob a forma de queijo e iogurtes, a baixa ingestão de carne vermelha e derivados, e o baixo-moderado consumo de vinho às refeições, especialmente o tinto. Acresce-se a escolha sazonal dos alimentos, locais e frescos ${ }^{24}$.

Estudos recentes mostram que o modelo alimentar mediterrânico, induz importantes modificações nos factores de risco cardiovascular e nos marcadores inflamatórios. Diminui a incidência e o desenvolvimento de doenças como obesidade, doenças metabólicas, cardiovasculares e neurodegenerativas e mesmo o cancro. As suas propriedades anti-oxidantes e anti-inflamatórias

22 Roda da Alimentação Mediterrânica (http://nutrimento.pt/cartazes/a-roda-da-alimentacao-mediterranica/).

${ }^{23}$ Altomare 2013: 449-457; Bonaccio 2012: 401-404.

${ }^{24}$ Estruch 2013: 1163-1166. 
actuam na longevidade e retardam o desenvolvimento de doenças crónicas associadas ao envelhecimento ${ }^{25}$.

Os países nórdicos têm vindo a adoptar este modelo saudável, substituindo o seu padrão alimentar, a dieta ocidental rica em gordura animal, açúcar e refeições processadas. Mas, enquanto os países do Norte da Europa têm reconhecido os benefícios da dieta mediterrânica, os povos mediterrânicos têm modificado os seus hábitos; é a ocidentalização da sua sociedade com as fast-food, as refeições processadas ricas em açúcar e gordura animal e pobres em legumes, vegetais, fruta e cereais, o beber episódico excessivo e a vida sedentária. Como interpretar esta mudança dos hábitos alimentares saudáveis para os não-saudáveis? Alterações sociais, globalização e migração, preço mais elevado dos alimentos saudáveis e condições sócio-económicas da população, poderão ser factores condicionantes. Há, de facto, uma associação entre a pobreza e a obesidade parcialmente explicada pelo baixo custo de alimentos altamente calóricos, reforçados pela palatabilidade do açúcar e das gorduras, reforços para a persistência da fome hedónica ${ }^{26}$.

O vinho está presente na dieta mediterrânica tanto como a bebida de eleição à refeição, neste caso o vinho tinto, como na culinária para fazer marinadas e temperos, sendo mais utilizado, aqui, o vinho branco.

Sobressai, como benéfica, a forma mediterrânica de beber vinho: o uso baixo-moderado, regular e à refeição, particularmente do vinho tinto que, como vimos, se associa a benefícios cardiovasculares e na diabetes, entre outros.

Mas importa não isolar os diversos constituintes da dieta mediterrânica. A dieta é rica na sua diversidade permitindo comer de tudo mas respeitando a quantidade e a frequência da ingestão dos mesmos. Na natureza não há um único alimento que seja nutricionalmente completo pelo que é necessário variar as escolhas e combinar apropriadamente os alimentos dos diferentes grupos. Eliminar um ou outro componente da dieta poderá destabilizar o seu efeito benéfico, pois há uma interacção sinergística e efeitos cumulativos entre os diversos alimentos e nutrientes ${ }^{27}$.

Os mecanismos subjacentes aos efeitos benéficos da dieta mediterrânica não são completamente conhecidos, mas a riqueza dos alimentos e do vinho em compostos anti-oxidantes e anti-inflamatórios e com efeitos na expressão de genes pró-aterogénicos, poderão explicar, pelo menos em parte, os seus benefícios na saúde ${ }^{28}$.

\footnotetext{
${ }^{25}$ Di Daniele 2017: 8947-8979; Martinez-Gonzalés 2015: 50-60.

26 Bonaccio 2012: 401-404.

Altomare 2013: 449-457.

28 Martinez-Gonzalés 2015: 50-60.
} 
Mais ainda, e como dissemos, a dieta mediterrânica não se limita a consumir uma dieta específica típica da região; é mais uma forma de filosofia de vida que não só nos indica o que comer mas também o que e como beber.

A ingestão regular de doses baixas-moderadas de vinho é um componente essencial da pirâmide alimentar mediterrânica, efectivo na prevenção primária e secundária de várias doenças crónicas, como anteriormente referido. $\mathrm{O}$ modelo saudável de alimentação envolve uma forma mediterrânica de beber, regular, moderada e preferencialmente à refeição (e não excessiva e episódica), e mais frequentemente o vinho tinto que tem assumido um papel de relevo pelo facto de conter uma mistura de substâncias bioactivas com repercussões benéficas no organismo.

Não é ainda claro qual dos componentes da dieta mediterrânica é o mais benéfico. Mas o uso preferencial do azeite, o consumo elevado de verduras e o modelo de ingestão de vinho são candidatos de relevo.

\section{Conclusão}

O vinho faz parte da História da Humanidade e da História da Medicina e é parte integrante do padrão alimentar mediterrânico. Consumi-lo de forma moderada ou excessiva pode significar a diferença entre remédio e veneno, entre prevenir e aumentar a mortalidade.

O consumo adequado de vinho alimenta o prazer, cuida do corpo, aumenta a longevidade. Um copo de vinho pode ser, simultaneamente, prazer e relaxamento e influenciar o humor e a sociabilidade, importantes ingredientes do bem-estar. Mas pode ainda ter, apesar das múltiplas dúvidas, efeitos benéficos em várias doenças como as cardiovasculares e a diabetes e possivelmente, também, nas doenças cancerígenas e na longevidade.

O conhecimento das qualidades e das propriedades preventivas e curativas do consumo baixo-moderado de vinho e das consequências do seu excessivo consumo é bem diferente do conhecimento de outrora, mas Hipócrates (460-370 a.C.) mantém-se no presente ${ }^{29}$ :

O vinho é uma bebida substancialmente maravilhosa apropriada ao homem, na saúde e na doença, se o administrarmos na justa medida, segundo a constituição de cada um.

\footnotetext{
${ }^{29}$ Padrões Culturais Editora 2005: 97.
} 


\section{REFERENCIAS}

Altomare, R., Cacciabaudo, F., Damiano, G., Palumbo, V. D., Giovialle, M. C., Bellavia, M., Tomasello, G., Lo Monte, A. I. (2013), “The mediterranean diet: a history of health", Iranian J Publ Health 42: 449-457.

Arranz, S., Chiva-Blanch, G., Valderas-Martinez, P., Medina-Remón, A., LamuelaRaventos, R. M., Estruch, R. (2012), "Wine, beer, alcohol and polyphenols on cardiovascular disease and cancer", Nutrients 4: 759-781.

Artero, A., Artero, A., Tarin, J. J., Cano, A. (2015), “The impact of moderate wine consumption on health", Maturitas 80: 3-13.

Bonaccio, .M, Iacoviello, L., Gaetano, G. (2012), “The mediterranean diet: the reasons for success", Thromb Res 129: 401-404.

Cueva, C., Gil-Sánchez, I., Ayuda-Durán, B., González-Manzano, S., GonzálezParamás, A. M., Santos-Buelga, C., Bartolomé, B., Moreno-Arribas, M. V. (2017), "An Integrated View of the Effects of Wine Polyphenols and Their Relevant Metabolites on Gut and Host Health", Molecules 22.1: 99, doi: 10.3390/ molecules22010099.

Dalby, A. (2003), Food in the Ancient World. From A to Z. London and New York, 350-352.

Di Daniele, N., Noce, A., Vidiri, M. F., Moriconi, E., Marrone, G., AnnicchiaricoPetruzzelli, M., D’Urso, G., Tesauro, M., Rovella, V., De Lorenzo, A. (2017). "Impact of Mediterranean diet on metabolic syndrome, cancer and longevity", Oncotarget 8: 8947-8979.

Estruch, R., Salas-Salvadó, J. (2013), “Towards an even healthier mediterranean diet”, Nutr Metab Eं Cardiovasc Dis 23: 1163-1166.

Klatsky, A. L. (2015), “Alcohol and cardiovascular diseases: where do we stand today?”, J Intern Med 278.3: 238-250, doi:10.1111/joim.12390.

Martinez-Gonzaléz, M. A., Salas-Salvadó, J., Estruch, R., Corella, D., Fitó, M., Ros, E. (2015), "Benefits of the mediterranean diet: insights from the PREDIMED study”, Progress in Cardiovasc Dis 58: 50-60.

O’Keefe, J. H., Bhatti, S. K., Bajwa, A., DiNicolantonio, J. J., Lavie, C. J. (2014), "Alcohol and cardiovascular health: the dose makes the poison... or the remedy", Mayo Clin Proc 89: 382-393.

Padrões Culturais Editora (2005,1. a ed.), O vinho como fármaco na sociedade-colectânea de excertos diversos, Rolo \& Filhos, Artes Gráficas, Lisboa.

Roda da Alimentação Mediterrânica, Disponível em: <http://nutrimento.pt/cartazes/aroda-da-alimentacao-mediterranica>.

Soares, C. (2014), “Pão e Vinho sobre a mesa: um 'clássico’ da alimentação Portuguesa”, in C. Soares, I. C. Macedo (eds.), Ensaios sobre o Património Alimentar Lusobrasileiro. Imprensa da Universidade de Coimbra-Annablume, Série DIAITA: Scripta \& Realia, Coimbra, 17-50.

Vilahur, G., Casani, L., Mendieta, G., Lamuela-Raventos, R. M., Estruch, R., Badimon, L. (2014), "Beer elicits vasculoprotective effects through Akt/eNOS activation", Eur J Clin Invest 44: 1177-1188.

Xiang, L., Xiao, L., Wang, Y., Li, H., Huang, Z., He, X. (2014), "Health benefits of wine: don't expect resveratrol too much", Food Chem 156: 258-263. 